\title{
- Pautas para el Diseño de Partes Metálicas Resistentes al Desgaste
}

* Luis Sampén A.

\section{RESUMEN}

El presente artículo analiza los mecanismos y principios del desgaste de materiales metálicos a fin de establecer pautas para el diseño adecuado de partes metálicas.

Palabras Claves: Desgaste metálico. Resistencia de materiales. Diseño de partes metálicas.

\section{ABSTRACT}

The present article analyses the mechanisms and principles of metal materials wearing, in order to set some guidelines for a suitable metal spare parts design.

Key Words: Metal wearing. Material Hard-Wearing. Metal spare parts design.

\section{INTRODUCCIÓN}

El proceso de desgaste, puede definirse como una pérdida de material de la interfase de dos cuerpos, cuando se les ajusta a un movimiento relativo bajo la acción de una fuerza. En general, los sistemas de ingeniería implican el movimiento relativo entre componentes fabricados a partir de metales y no metales, y se han identificado seis tipos principales de desgaste, como sigue:
1. Desgaste por adherencia.
2. Desgaste por abrasión.
3. Desgaste por ludimiento.
4. Desgaste por fatiga.
5. Desgaste por erosión.
6. Desgaste por cavitación.

Aquí se estudian brevemente los principios que se aplican para disminuirlo. Sin embargo, es necesario definir el mecanismo de estas seis formas de desgaste.

\section{DESGASTE POR ADHERENCIA}

En este caso, el movimiento relativo puede ser deslizamiento unidireccional o de vaivén, o bien la interacción ocurre bajo carga en un contacto oscilatorio de pequeña amplitud. Se sabe que los picos superficiales que coinciden fluyen plásticamente y forman fuertes uniones endurecidas por el trabajado. A medida que estas se rompen bajo la tracción tangencial impuesta, los sólidos van perdiendo material.

\section{DESGASTE POR ABRASIÓN}

Las partículas abrasivas producidas por los residuos del desgaste o por partículas extrañas de arena y polvo circundante permanecen atrapadas en la superficie deslizante y eliminan material principalmente por formación de surcos.

\section{DESGASTE POR LUDIMIENTO}

El desgaste por ludimiento aparece como resultado del movimiento oscilatorio de dos superficies en contacto, como sucede en máquinas donde existe vibración entre las partes.

* Docente del Departamento de Diseño y Tecnología.

Facultad de Ingeniería Industrial, UNMSM

E-mail: 1sampena@unmsm.edu.pe 


\section{DESGASTE POR FATIGA}

Es probable que el modo predominante de la mayoría de los tipos de desgaste sea por desprendimiento de material de la superficie por fatiga, ya sea que la naturaleza del movimiento sea unidireccional o de vaivén. Clasificar un tipo particular de falla como desgaste por fatiga puede ser confuso. Sin embargo, a fin de hacer un clasificación, el término desgaste por fatiga se reserva para identificar la falla de contactos lubricados en casos como los rodamientos de bolas o rodillo, engranes, levas y mecanismos impulsores de fricción. La pérdida de material es por desprendimiento de capas superficiales y por picaduras, como en los engranes.

Se piensa que las grietas por fatiga aparecen debajo de la superficie en un punto en que el esfuerzo cortante es máximo. Obviamente, puede lograrse un mejoría en la vida de estos elementos, si trabaja a un carga de contacto baja y el método más preferido en la industria es producir componentes con la profundidad óptima de capa endurecida junto con un buen acabado superficial. El propósito de esta capa externa dura tal como se obtiene por carburación, nitruración o sulfurización es proporcionar una superficie con un alto límite de resistencia en una región vulnerable a la iniciación de grietas.

\section{DESGASTE POR EROSIÓN}

El desgaste por erosión se define como el proceso de eliminación de metal provocado por la incidencia de partículas sólidas sobre una superficie. El desgaste por erosión es deliberado como en el caso de la limpieza de piezas de colada o cascos de barco por medio de chorros de arena, pero en ocasiones se produce una pérdida destructiva y costosa de material como en el caso de las hélices de turbinas de gas o los refractarios en hornos de arco eléctrico. El grado de desgaste tiene relación con el ángulo de incidencia de la partícula respecto de la superficie. Los materiales dúctiles parecen deformarse y posiblemente se endurezcan cuando se les golpea en forma perpendicular, pero a un ángulo crítico de aproximadamente $20^{\circ}$, el metal se elimina por una acción de corte. Los materiales frágiles fallan por agrietamiento de las superficies cuando la fuerza de impacto es normal. Se deduce que un componente dúctil finalmente se endurecerá por trabajado y fallará como si fuera frágil.

Se han hecho intentos de manipular el ángulo de incidencia por modificación del diseño del componente en sí o por protección de las superficies con un material como el hule. El control del ángulo de incidencia, es una situación industrial que no es fácil y es pertinente especular si las superficies deben recubrirse con medios que se puedan restaurar si las superficies deben recubrirse con medios que se puedan restaurar fácilmente como un procedimiento programado de mantenimiento.

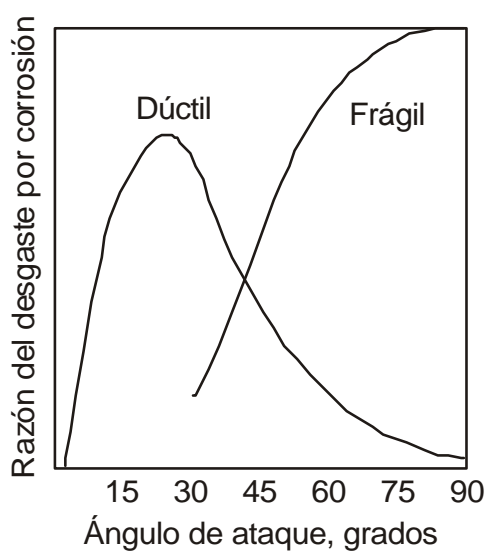

Figura 1. Razón del desgaste por erosión en sólidos dúctiles y frágiles, en relación con el ángulo de ataque de las partículas incidentes.

\section{EROSIÓN POR CAVITACIÓN}

La erosión por cavitación se presenta cuando un sólido se mueve a alta velocidad en un medio líquido, siendo un caso típico el de las hélices de los barcos. Mientras que el desgaste por erosión implica una acción meramente mecánica, se cree que la erosión por cavitación está relacionada con la formación de burbujas dentro del medio líquido, a través del cual pasa el componente sólido. La erosión por cavitación se produce también en los rodamientos lubricados.

Se ha demostrado que la fuerza del impacto causado excede el punto de fluencia de la mayoría de los metales.

Quizá los metales más efectivos sean los duros que tienen una elevada resistencia de fluencia, o que se endurecen por trabajo en el servicio. Los materiales más adecuados son la estelita y el acero inoxidable 18/8. El bronce al manganeso colado es un material preferido para hélices marinas. Tiene una resistencia inferior a la erosión por cavitación que la estelita o que el acero inoxidable, pero es resistente a la corrosión marina y se ha demostrado que la corrosión agrava la erosión por cavitación. 
Además, de recubrir un sustrato metálico con revestimientos de cromo duro, con frecuencia se utiliza una capa elástica que absorbe las elevadas fuerzas de impacto. Como ejemplo de estos últimos materiales están el poliuretano y el neopreno, entre otros.

\section{DISEÑO PARA PROPORCIONAR RESISTENCIA AL DESGASTE POR ADHERENCIA Y POR ABRASIÓN}

Tanto el desgaste por adherencia como el desgaste por abrasión se puede considerar en la ingeniería como la forma más común de desgaste. Siempre que dos superficies entran en contacto existe la probabilidad de adherencia en puntos favorables de la interfase seguida, probablemente, por el desgaste. Es poco probable que la interfase esté libre de areniscas y residuos de desgaste, de manera que en tales casos el mecanismo de desgaste, como en los rodamientos lisos y las camisas de cilindros, es seguramente una combinación de adherencia y abrasión.

Al diseñar partes a prueba de desgaste, no es recomendable seleccionar un par de metales que presenten solubilidad mutua. De este modo, acero que se deslice sobre acero no es un proposición lógica aunque se ha tenido éxito de deslizar hierro colocado sobre sí mismo, quizá por la presencia de grafito. Tanto las leyes del desgaste por adherencia, como las del desgaste por abrasión, muestran que entre más duro sea un componente, más resistente será el desgaste y esto se confirma en la práctica. Una de las dificultades con los materiales excesivamente duros es que son susceptibles de sufrir fallas por fractura y, para evitar las fallas mecánicas, el componente debe ser tenaz lo que es característico de los materiales blandos y dúctiles; por lo tanto, para mantener la tenacidad parece necesario sacrificar dureza. Sin embargo, mediante tratamientos superficiales y térmicos de los componentes es posible alcanzar un núcleo tenaz con una superficie dura.

En general, si no existe movimiento oscilatorio, corrosión ni temperatura elevada, se pueden aplicar los siguientes principios para seleccionar aceros:

a. Fuerza de impacto baja: se utilizan carburos duros en la microestructura 0 se nitrura 0 carburiza el acero. También pueden emplearse hierros y aceros martensíicos.

b. Fuerza de impacto alto: el criterio recomendable es utilizar aceros austeníticos, inoxidables o el acero Hadfield al manganeso.

Se han hecho tres clasificaciones amplias de los métodos tribológicos para disminuir el desgaste, a saber, el uso de una capa protectora y los principios de conversión y diversitificación.

\section{Capa Protectora}

Sobre todas las superficies se forma inevitablemente una capa de óxidos o gases sorbidos, pero sin ésta, muchos casos de deslizamiento en seco, como el caso de las vías de ferrocarril, no resistirían el servicio por un periodo aceptable. Invariablemente, en la mayoría de las situaciones se utilizan lubricantes líquidos o sólidos.

En la actualidad hay un renovado interés por evaluar los diversos tipos de tratamientos superficiales antes de poner en servicio los componentes, con el propósito de lograr resistencia al desgaste. Hablando en forma general el tratamiento puede clasificarse en dos tipos:

a. Depósito de un metal o un no metal sobre el componente.

b. Tratamiento de difusión como el carburizado o nitruración.

Como ejemplos de depósito están los fosfatos sobre hierro colado y acero de estaño sobre aluminio. Estos tienen vidas limitadas y protegen las superficies del daño severo durante el arranque inicial. Otras formas de tratamiento por difusión son, la sulfurización, cromación y los recubrimientos electrolíicos. La metalización y aspersión del metal o las técnicas de recubrimiento con superficies duras, como el uso de revestimientos soldados, también proporcionan películas resistentes al desgaste.

\section{Principio de Conversión}

El principio de conversión permite el desgaste de una parte del sistema para ofrecer protección a otros componentes más importantes. Un ejemplo es el uso de anillos para pistones de hierro colado que, al desgastarse rápidamente, evitan el rayado de la camisa del cilindro.

\section{Principio de Diversificación}

El componente menos costoso, por ejemplo un cojinete liso, está diseñado para desgastarse a fin de proteger a tramo de flecha, más caro, del daño superficial y el desgaste. En las chumaceras, los metales blancos, que contienen estaño, plomo, cobre y antimonio, proporcionan una superficie blanda de deslizamiento. Sin embargo, si se incrementa la fuerza y la velocidad, será necesario utilizar superficies más duras en los cojinetes, como las que proporcionan los bronces.

\section{IMPORTANCIA DEL DESGASTE}

El desgaste de las partes implica reposición y esto en sí es costoso. 


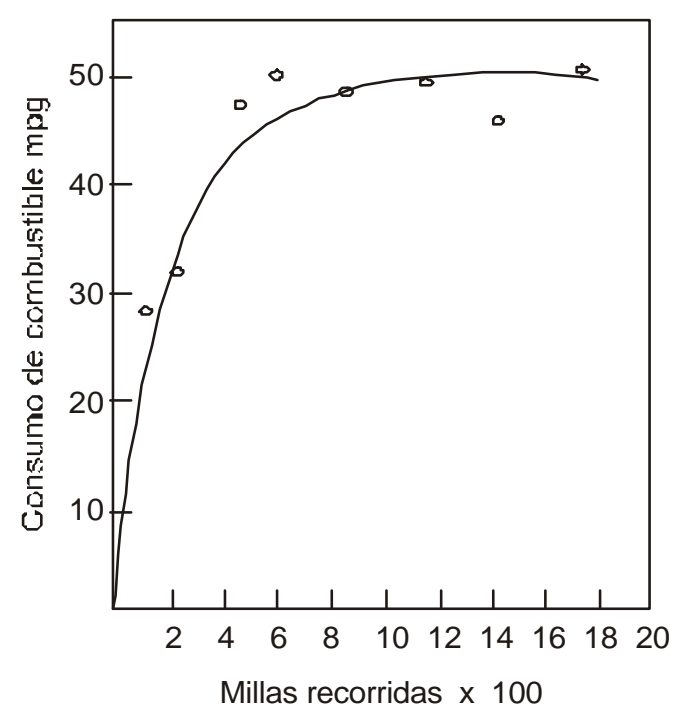

Figura 2. Consumo de combustible de un automóvil nuevo por distancia de deslizamiento. Es notable el alto consumo de combustible durante la etapa de arranque inicial.

Mas, aun, las superficies desgastadas hacen que se pierda la precisión y merma la eficiencia. Por lo tanto, deben hacerse todo lo posible para diseñar con una cantidad de desgaste mínima.

Se tiene suficientes datos para demostrar que con una fuerza característica, aunque dependiendo de las propiedades de metal, se produce una rápida transición que da lugar a un desgaste severo a partir del régimen precedente de desgaste moderado. Es indispensable que las partes móviles de la maquinaria sean diseñadas de tal manera que a la fuerza de contacto no exceda las condiciones que determina el régimen de desgaste moderado. A la larga no es más costoso utilizar materiales con alto esfuerzo de fluencia para permitir cargas pesadas en el servicio.

Si bien se hace todo por disminuir el desgaste en el estado estacionario, durante el arranque inicial de las máquinas nuevas se deben eliminar las capas superficiales. El proceso de arranque inicial probablemente implica la creación de una capa subcutánea deformada que será lo bastante dura para resistir el estado estable del desgaste, pero los picos superficiales, deben desgastarse y deformarse para mejorar el ajuste de las partes en interacción. El hecho que en esta etapa se realice un trabajo mecánico adicional que interviene en la deformación y el desgaste, se aprecia en la Figura 2 que muestra el alto consumo de combustible durante el arranque inicial de un automóvil nuevo. Sin embargo, es difícil pronosticar la razón de desgaste porque depende no sólo de la carga y la velocidad de la superficie, sino también de otros factores que no siempre es posible evitar. Siempre hay presencia de abrasivos extraños o de residuos, que se forman por interacción y como se sabe, los abrasivos incrementan la razón de desgaste. La elevada velocidad superficial es benéfica, porque eleva la temperatura interfacial, lo que facilita la formación de películas de óxido.

En el caso de motores de gasolina, se produce aproximadamente un galón de agua por cada galón de combustible quemado. Los productos de combustión contienen óxidos de carbono, azufre y pequeñas cantidades de compuestos de bromo y cloro. Si la temperatura de la pared del motor es baja, el agua y los productos de combustión ácidos corrosivos para la pared del cilindro y para los anillos de los pistones. El desgaste se acentúa como se muestra en la Figura 3. No es de sorprender que no se haya propuesto una sola ley del desgaste, que se pueda aplicar con confianza para predecir la razón de desgaste de los componentes de interacción. Una razón importante es que, en común con muchos sistemas tecnológicos, la mayoría de las situaciones tribológicas actúan bajo la acción combinada de diversas variables. Por ejemplo, la abrasión puede estar superpuesta a una situación que se diseñó en primer término para el desgaste por adherencia y el problema se agrava si el sistema funciona en un entorno hostil. Por lo tanto, es indispensable que al diseñar partes a prueba de desgaste, se identifiquen las condiciones de servicio de los componentes. Sin embargo, para predecir las razones de desgaste, debe existir una continua inversión en investigación encaminada a obtener información sobre los mecanismos de los diversos tipos de desgaste en superficies que interactúan al estar sujetas a movimiento relativo.

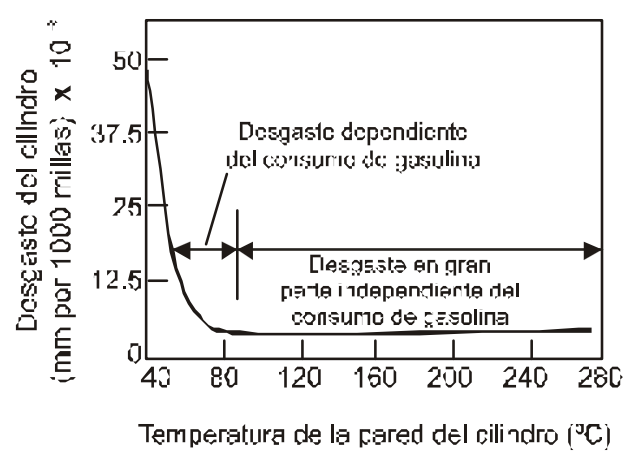

Figura 3. Desgaste de un motor en función de la temperatura de la pared del cilindro.

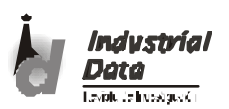

diciembre 2003
Notas Científicas 


\section{PROCEDIMIENTO PARA LA SELECCIÓN DE MATERIALES}

La importancia de la selección de materiales para la resistencia a desgaste requiere un estudio de los detalles del desgaste al fin de determinar cuál de las propiedades convencionales del material puede resistirlo mejor. Los mejores instrumentos que se pueden emplear son los microscopios, y la fotografía si es posible. El microscopio más útil es el tipo con estereoscopia y con lente "zoom" con un poder de amplificación de $1 x$ a 7x, con oculares de $5 \times 0$ 10x y una luz externa movible de 100 watts. La observación estereoscópica de la perspectiva de las formas de los detalles de la superficie, como ranuras, pliegues, escamas, etc. El otro instrumento de suma utilidad es el microscopio electrónico explorador (con "escaner") reflejante (SEM). Un analista debe emplear el SEM junto con microscopios ópticos, pues cada uno produce fotografías muy diferentes de las superficies. A menudo, las observaciones más útiles con el SEM son las de baja amplificación, entre 20x y 200x. La ventaja del SEM es que se pueden ver superficies muy ásperas sin que las regiones altas y las bajas queden fuera de foco, como ocurre con los microscopios ópticos. El problema principal es que el SEM sólo recibe muestras pequeñas; por ejemplo, de $1 \frac{1}{2}$ in $(12.5 \mathrm{~mm})$ de espesor y 2 in (50 $\mathrm{mm}$ ) de diámetro y las superficies deben estar limpias porque en el SEM se requiere un vacío de alrededor de $10^{-5}$ torr.

Para efectuar un análisis más detallado de la composición química de la superficie, se puede utilizar un SEM con aditamento de dispersión de rayos $X$. También se pueden emplear los microscopios ópticos metalúrgicos, pero no con el modo de campo brillante con luz reflejada.

En ocasiones es útil obtener un perfil de la configuración de superficies gastadas, lo cual se puede hacer con el estilete empleado en la medición convencional de acabado de superficie, pero debe estar conectado con un registrador de gráficas movible. Lo que es más útil es la forma en vez del valor numérico del acabado de la superficie. Los trazos se deben hacer en diversos lugares y sentidos durante el avance del desgaste, si es posible. Una precaución importante para el examen de los datos de la tira de gráfica es que la representación de la altura de las configuraciones de la superficie, a veces, está amplificada entre
10 y 1000 veces más que la dimensión horizontal. Con ello, se hacen bocetos de superficies con crestas o picos muy pronunciados.

Una vez examinado un sistema de desgaste, el diseñador puede consultar tablas guías para determinar las propiedades de los materiales que resisten al desgaste y hacer una selección adecuada.

\section{CONCLUSIONES}

El desgaste es importante para el diseño de elementos de máquinas.

El conocimineto los diferentes tipos de desgaste, permite diseñar adecuadamente las partes que estarán en contacto a fin de controlar el desgaste mediante modificaciones al movimiento, cargas de impacto 0 aplicación de los principios de conversión y diversificación.

Las investigaciones futuras deben concentrarse en el mecanismo del desgaste moderado y del desgaste en el arranque inicial para proporcionar información a los ingenieros de diseño.

\section{BIBLIOGRAFÍA}

1. Bayer, R.G. (1988). Principales of Tribology. $2^{\circ}$ Ed. Edit. MacMillan Press, England.

2. Lipson, C. (1975). Wear Considerations in Design. $1^{\circ} \mathrm{Ed}$. Edit. Prentice - Hall, USA.

3. Ludema, K. C. (1981). Selecting Materials for Wear Resistance, in third International Conference on Wear of Materials, ASME, San Francisco.

4. Sarkar, A.D. (1998). Desgaste de Metales. $1^{0}$ Ed. Edit Limusa, México.

5. Tabor, D. (1995). The Hardness of Metals, Oxford University Press. 